\title{
THE IMPRINT OF RADIAL MIGRATION ON THE VERTICAL STRUCTURE OF GALAXY DISKS
}

\author{
Carlos Vera-Ciro ${ }^{1,2}$, Elena D'Onghia ${ }^{2,4}$, and Julio F. Navarro ${ }^{3,5}$ \\ ${ }^{1}$ Departamento de Ciencias Básicas, Universidad de Medellín, Cra 87 N 30-65, Medellín, Colombia; cvera@udem.edu.co \\ ${ }^{2}$ Department of Astronomy, University of Wisconsin, 475 N. Charter Street, Madison, WI 53076, USA \\ ${ }^{3}$ Department of Physics and Astronomy, University of Victoria, Victoria, BC, V8P 5C2, Canada \\ Received 2016 May 9; revised 2016 September 14; accepted 2016 September 16; published 2016 December 6
}

\begin{abstract}
We use numerical simulations to examine the effects of radial migration on the vertical structure of galaxy disks. The simulations follow three exponential disks of different mass but similar circular velocity, radial scalelength, and (constant) scale height. The disks develop different non-axisymmetric patterns, ranging from feeble, long-lived multiple arms to strong, rapidly evolving few-armed spirals. These fluctuations induce radial migration through secular changes in the angular momentum of disk particles, mixing the disk radially and blurring pre-existing gradients. Migration primarily affects stars with small vertical excursions, regardless of spiral pattern. This "provenance bias" largely determines the vertical structure of migrating stars: inward migrators thin down as they move in, whereas outward migrators do not thicken up but rather preserve the disk scale height at their destination. Migrators of equal birth radius thus develop a strong scale-height gradient, not by flaring out as commonly assumed, but by thinning down as they spread inward. Similar gradients have been observed for low- $[\alpha / \mathrm{Fe}]$ monoabundance populations (MAPs) in the Galaxy, but our results argue against interpreting them as a consequence of radial migration. This is because outward migration does not lead to thickening, implying that the maximum scale height of any population should reflect its value at birth. In contrast, Galactic MAPs have scale heights that increase monotonically outward, reaching values that greatly exceed those at their presumed birth radii. Given the strong vertical bias affecting migration, a proper assessment of the importance of radial migration in the Galaxy should take carefully into account the strong radial dependence of the scale heights of the various stellar populations.
\end{abstract}

Key words: galaxies: kinematics and dynamics - Galaxy: disk - Galaxy: evolution - stars: kinematics and dynamics

\section{INTRODUCTION}

Non-axisymmetric patterns in disk galaxies have long been known to drive secular changes in the energy and angular momentum of disk stars (e.g., Lynden-Bell \& Kalnajs 1972). Angular momentum changes are generally accompanied by radial shifts in the average galactocentric distance of a star. Although such changes usually lead to increased random motions and a gradual increase in the velocity dispersion of the disk, Sellwood \& Binney (2002) demonstrated, in an influential paper, that stars can also vary their angular momenta while preserving their circularities if they are near corotation of fluctuating spiral patterns, a process that leads to limited disk heating and is usually referred to as radial migration.

This realization has led to new insights regarding the interpretation of a number of properties of galaxy disks, including (1) the (weak) correlation between age and metallicity in the solar neighborhood (Edvardsson et al. 1993; Haywood 2008; Bergemann et al. 2014); (2) the upturn of the mean age in the outer parts of disks (Bakos et al. 2008; Roškar et al. 2008); (3) the formation of the Galactic thick disk (Schönrich \& Binney 2009; Loebman et al. 2011); and (4) the radial dependence of the metallicity distribution function in the Galactic disk (Hayden et al. 2015; Loebman et al. 2016; Kawata et al. 2016; Martinez-Medina et al. 2016; Miranda et al. 2016). Although there is a consensus that migration should somehow contribute to those trends, its actual importance in each particular case is still a matter of debate.

\footnotetext{
Alfred P. Sloan Fellow.

5 Senior CIfAR Fellow.
}

One example concerns the effect of migration on the vertical structure of the Galactic disk. Earlier work suggested that migration can thicken a galaxy disk by pushing stars from the inner regions (where the velocity dispersion is high) to the outskirts of the disk, where the surface density - and hence the vertical restoring force-is lower (e.g., Schönrich \& Binney 2009). Minchev et al. (2012), on the other hand, argued that migration does not necessarily lead to disk thickening, since outward migrators should conserve their vertical action and cool down as they move out (see also Solway et al. 2012; Roškar et al. 2013; Vera-Ciro \& D'Onghia 2016). The net effect on disk thickness will therefore depend on whether the migrating population is kinematically hotter or cooler than nonmigrating stars at their final destinations.

The vertical structure of migrators was also studied by VeraCiro et al. (2014), who noted that migrators are a heavily biased subset of stars with preferentially low vertical velocity dispersions. This "provenance bias" arises because stars with small vertical velocities spend more time near the disk plane, and thus couple more readily to non-axisymmetric perturbations. The resulting vertical structure of migrators is further complicated by the fact that the velocity dispersion of outward migrators generally decreases, whereas the opposite applies to inward migrators.

A potential application of these ideas to the Galactic disk is provided by recent results from the APOGEE survey. By dissecting the Milky Way stellar disk into distinct populations according to their $\alpha$ and $\mathrm{Fe}$ content, Bovy et al. (2016, hereafter B16) were able to confirm the presence of two chemically distinct populations differing in their $[\alpha / \mathrm{Fe}]$ ratios. 
This distinction provides an improved characterization of the traditional "thick" and "thin" disks of the Galaxy that does not rely on kinematics. Such purely chemical characterization is highly preferable, since, unlike kinematics, chemistry is a durable and stable property of a star that should faithfully reflect its local conditions at birth (see, e.g., Navarro et al. 2011).

An interesting property of low- $[\alpha / \mathrm{Fe}]$ stars (i.e., the traditional "thin disk") is that their spatial distribution varies smoothly and systematically with iron abundance. In particular, the surface density of stars of a mono-abundance population (MAP) of fixed $[\mathrm{Fe} / \mathrm{H}]$ peaks at some radius and decreases both inside and outside that radius. The "peak radius" increases with decreasing $[\mathrm{Fe} / \mathrm{H}]$, in a manner that defines the overall metallicity gradient of the disk.

More importantly for our current discussion, there are clear radial trends in the thickness of each MAP: they flare outward, a result that has been interpreted by B16 as an "essential test of the predictions of radial migration." This conclusion relies on a simple scenario where, broadly speaking, stars in each MAP share a similar birth radius-i.e., the "peak radius" of their radial distribution - and disperse through the Galaxy by the secular changes induced by spiral patterns, flaring outward in the process.

However, given the uncertainties in our understanding of the effects of radial migration cited earlier, this scenario seems premature. Here, we report on results of a set of numerical simulations of isolated stellar disks designed to test the role of radial migration in establishing radial gradients in scale height. The simulations were designed so that disks develop different spiral patterns, allowing us to assess the robustness of our conclusions regarding the specific nature of the spiral, such as a number of arms and the overall strength of the pattern. The numerical experiments here presented do not include gas, star formation, nor the inside-out growth of the stellar disk as, e.g., in Minchev et al. (2015). Although our models cannot capture the full complexity of the formation and evolution of the Milky Way disk, our methodology should be able to clarify how and whether migrators might be responsible for the radial dependence of the scale height of different MAPs.

This paper is organized as follows. Section 2 describes the numerical simulations while our main results are presented in Section 3. We end with a brief discussion and a summary of our main conclusions in Section 4.

\section{NUMERICAL SIMULATIONS}

\subsection{Galaxy Models}

The stellar disk in all of our simulations is described by an exponential surface density profile: $\Sigma(R)=M_{\mathrm{d}} /\left(2 \pi R_{\mathrm{d}}^{2}\right)$ $\exp ^{\left(-R / R_{\mathrm{d}}\right)}$, with $M_{\mathrm{d}}$ the total disk mass and $R_{\mathrm{d}}$ the disk scale length. The vertical distribution of disk stars follows an isothermal sheet with a radially constant scale height, $z_{\mathrm{d}}$. The 3D stellar density in the disk is then

$$
\rho_{\mathrm{d}}(R, z)=\Sigma(R) \zeta(z)=\frac{M_{\mathrm{d}}}{4 \pi z_{\mathrm{d}} R_{\mathrm{d}}^{2}} \operatorname{sech}^{2}\left(z / z_{\mathrm{d}}\right) \exp \left(-R / R_{\mathrm{d}}\right)
$$

The disk mass is sampled with $N=5 \times 10^{6}$ particles and is evolved in a dark matter halo modeled as a static Hernquist profile (Hernquist 1990). One of the simulations also includes a bulge modeled as a second Hernquist profile.
Table 1

Galaxy Model Parameters

\begin{tabular}{lccr}
\hline \hline Parameters & LD & HD & HD-MW \\
\hline$M_{\mathrm{d}}\left[10^{10} M_{\odot}\right]^{\mathrm{a}}$ & 1.91 & 4.00 & 4.00 \\
$R_{\mathrm{d}}[\mathrm{kpc}]^{\mathrm{b}}$ & 3.13 & 2.84 & 2.50 \\
$z_{\mathrm{d}} / R_{\mathrm{d}}{ }^{\mathrm{c}}$ & 0.10 & 0.10 & 0.15 \\
$M_{\text {halo }}\left[10^{12} M_{\odot}\right]^{\mathrm{d}}$ & 0.93 & 1.00 & 9.50 \\
$R_{\text {halo }}[\mathrm{kpc}]^{\mathrm{e}}$ & 29.78 & 25.77 & 130.0 \\
$M_{\text {bulge }}\left[10^{10} M_{\odot}\right]^{\mathrm{f}}$ & $\ldots$ & $\ldots$ & 1.40 \\
$R_{\text {bulge }}[\mathrm{kpc}]^{\mathrm{g}}$ & $\ldots$ & $\ldots$ & 0.35 \\
$f_{\text {disk }}\left(2.2 R_{\mathrm{d}}\right)^{\mathrm{h}}$ & 0.28 & 0.42 & 0.48 \\
\hline$t_{0}[\mathrm{Gyr}]^{\mathrm{i}}$ & 0.98 & 0.10 & 0.29 \\
$t_{1}[\mathrm{Gyr}]^{\mathrm{j}}$ & 3.42 & 0.88 & 0.98 \\
$t_{2}[\mathrm{Gyr}]^{\mathrm{j}}$ & 9.78 & 2.05 & 1.86 \\
\hline
\end{tabular}

Notes.

${ }^{\mathrm{a}}$ Disk mass.

${ }^{\mathrm{b}}$ Disk scale length.

${ }^{\mathrm{c}}$ Disk scale height.

${ }^{\mathrm{d}}$ Halo mass.

${ }^{\mathrm{e}}$ Halo scale length.

${ }^{\mathrm{f}}$ Bulge mass.

${ }^{\mathrm{g}}$ Bulge scale length.

${ }^{\mathrm{h}}$ Disk mass fraction within $2.2 R_{\mathrm{d}}$.

${ }^{\mathrm{i}}$ Initial time.

${ }^{\mathrm{j}}$ Times when radial velocity dispersion has increased by $10 \%-30 \%$ respectively.

We consider three different galaxy models, labeled LD ("light disk"), HD ("heavy disk"), and HD-MW ("Milky Waylike"). The structural parameters of each galaxy model are listed in Table 1.

The LD galaxy is the same model as presented by D'Onghia et al. (2013) and analyzed in detail by Vera-Ciro et al. (2014). It corresponds to a low-mass disk, where the spiral patterns are recurrent and relatively weak, but long-lived and characterized by multiple arms.

The HD model is a heavier-disk version of the LD galaxy, where the disk mass has been increased by a factor of two while keeping the halo parameters basically unchanged.

Finally, the model labeled HD-MW is designed to have a disk mass and a radial scalelength that are consistent with the Milky Way. The addition of a bulge and the adjustment of the halo parameters yields a flat circular velocity curve in good agreement with that of the Galaxy; i.e., $V_{\text {circ }} \sim 220 \mathrm{~km} \mathrm{~s}^{-1}$ roughly constant in the range of $0.5<R<14 \mathrm{kpc}$. In this case, the disk contributes $\approx 70 \%$ of the total circular speed at 2.2 scale lengths (Bovy et al. 2012).

The radial component of the disk stellar velocity dispersion, $\sigma_{R}=\sigma_{\theta}$, is assumed to scale proportionally with the vertical component, $\sigma_{z}: \sigma_{R}^{2}=f_{R} \sigma_{z}^{2}$, where $\sigma_{z}$ is specified by the chosen scale height, $z_{\mathrm{d}}$ (Hernquist 1993). The ratio $z_{\mathrm{d}} / R_{\mathrm{d}}$ is chosen to be in the range of $0.1-0.15$ for all disks. The factor $f_{R}$ is chosen so as to set a minimum value of the Toomre parameter $\left(Q=\sigma_{R} \kappa /(3.36 G \Sigma)\right)$ of unity at two disk scale lengths.

\subsection{The Runs}

The simulations were carried out with the parallel TreePM code GADGET-3. We only employ the tree-based gravity solver coupled to a static external potential to solve for the evolution of collisionless particles. Pairwise particle interactions are softened with a spline kernel (Hernquist \& Katz 1989) 

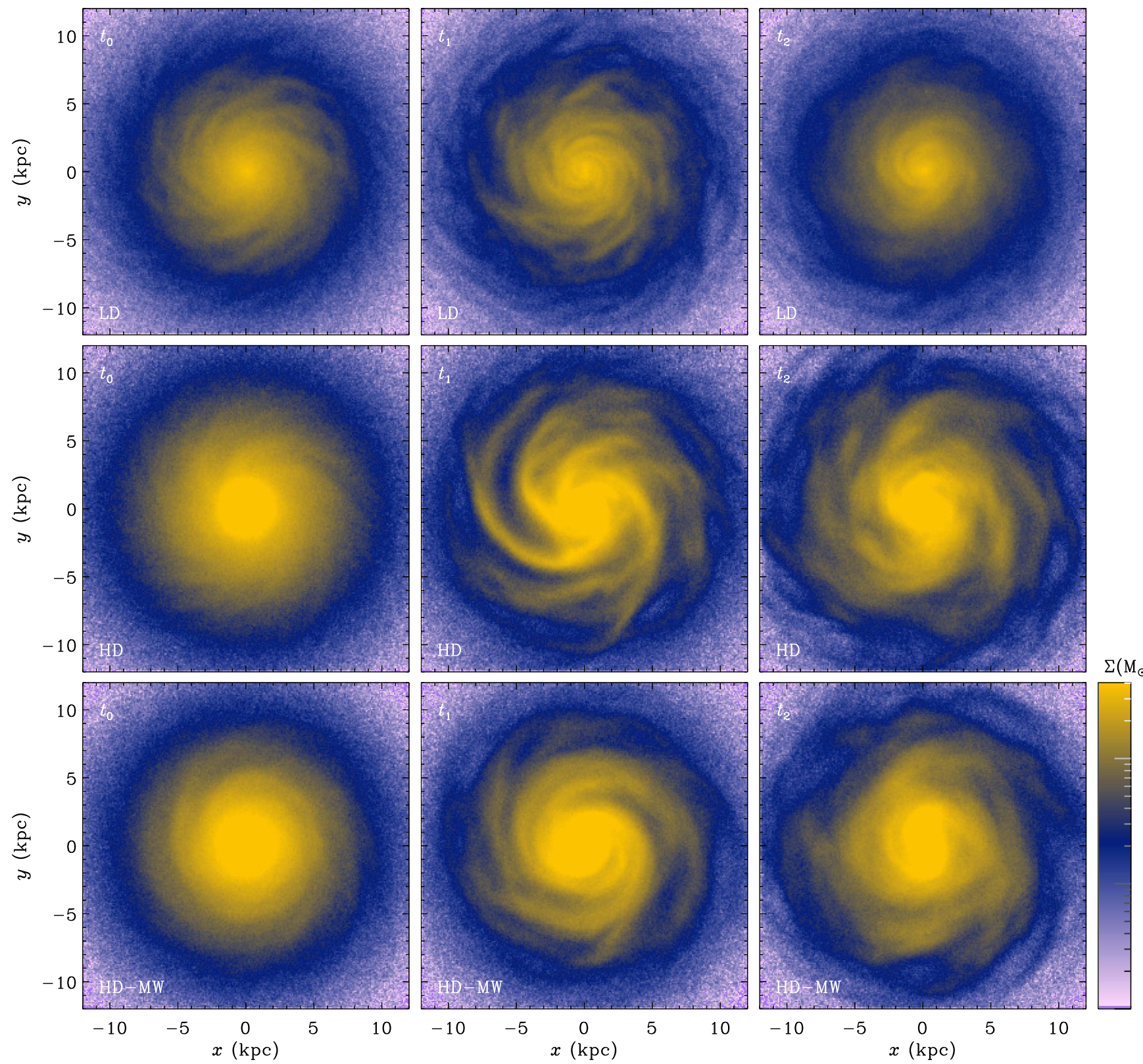

Figure 1. Face-on disk projected stellar density for the three simulated galaxies: the low-mass disk case LD (top panels), and the two heavy-disk models HD (middle panels) and HD-MW (bottom panels). Note the different spiral patterns in each case. The two heavy disks eventually develop a strong central bar. The structural parameters of the disks and characteristic times are listed in Table 1.

on scales $h_{s}$, so that forces are strictly Newtonian for particles separated by more than $2 h_{s}$. The resulting force is roughly equivalent to traditional Plummer-softening with scale length $\approx h_{s} / 2.8$. For our simulations, the gravitational softening length was set to $50 \mathrm{pc}$.

\subsection{Disk Evolution}

Because of their different mass and circular velocity profiles, each disk develops different spiral patterns over time, as shown in Figure 1. The LD model may be described as a flocculent spiral with multiple arms, whereas the two heavy disks show stronger patterns with fewer arms that evolve relatively quickly into a central bar. The non-axisymmetric perturbations gradually heat the disk in all cases, but at a much faster rate for the case of the heavier disks than for the LD model.
In an attempt to compare the three models at comparable stages of evolution we select three characteristic times for analysis; (1) an initial time, $t_{0}$, before the spiral patterns develop and defined so that the rms potential fluctuations on the disk plane in the radial range of $3<R / \mathrm{kpc}<8$ is small (i.e., $\sigma_{\Phi} \sim 2.5 \times 10^{-4}$ ); (2) an intermediate time, $t_{1}$, when the radial velocity dispersion in that same radial range has increased by $10 \%$; and (3) a final time, $t_{2}$, when $\sigma_{R}$ has increased by $30 \%$ since $t_{0}$. We list these times (in gigayears) in Table 1. Note that it takes many more half-mass disk rotations to heat up the LD model (24/87) than the other two (10/26 and $13 / 30$ for HD and HD-MW, respectively).

Figure 2 shows the surface density profiles, as well as the circular velocity and velocity dispersion profiles at those three times for all disks. Aside from the development of a central $(\sim 1$ kpc) bar in the case of the heavy disks, the most noticeable 

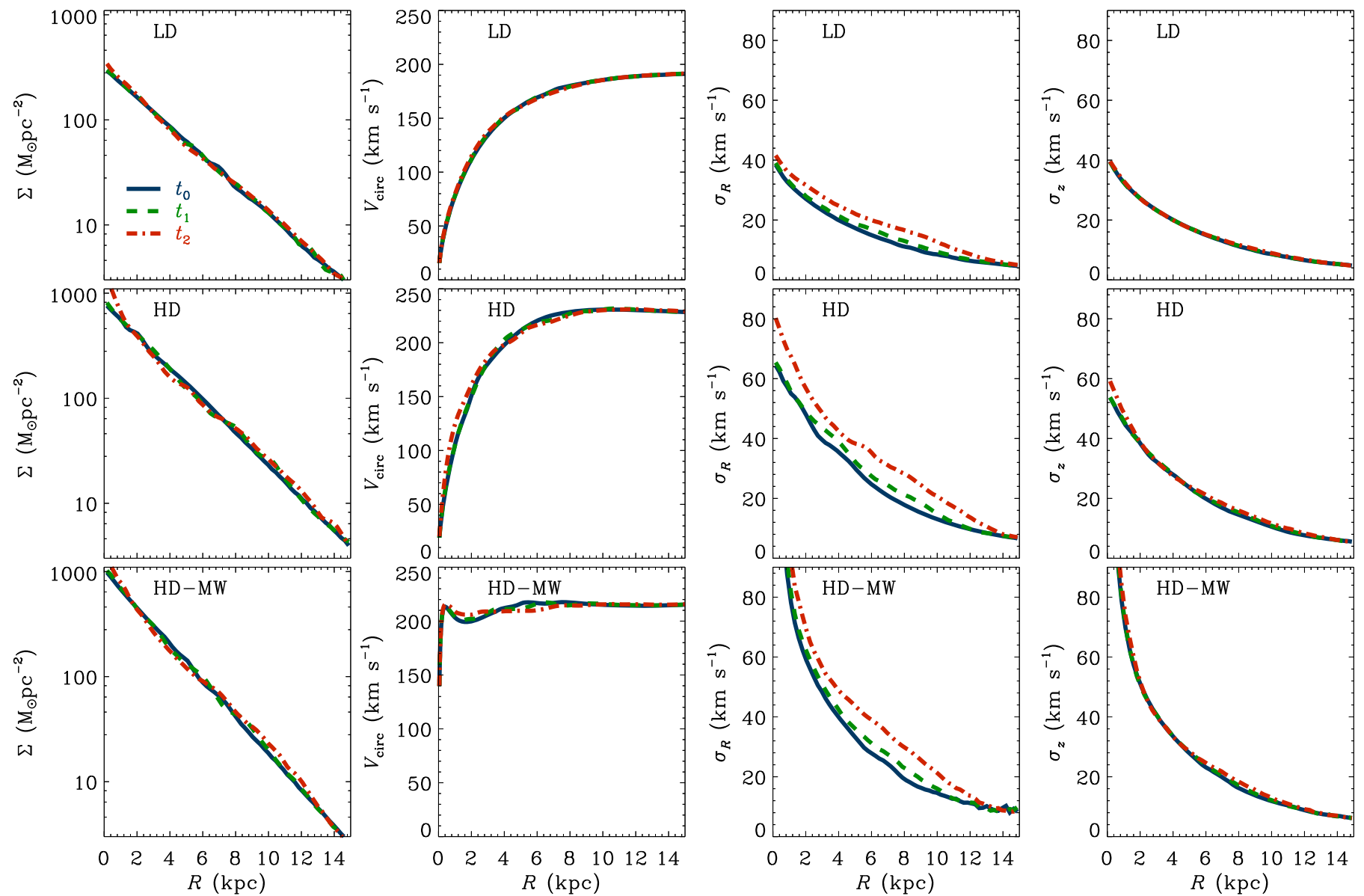

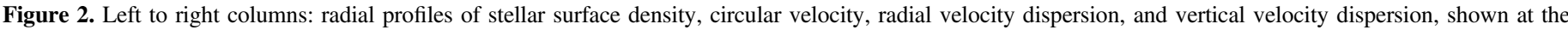

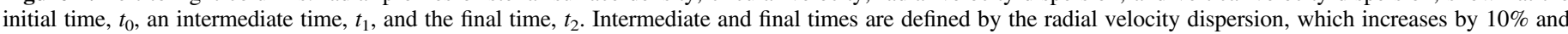
$30 \%$ since $t_{0}$. All times are listed in Table 1 .

change from $t_{0}$ to $t_{2}$ is in the radial velocity dispersion, which, by construction, grows by $30 \%$ in all cases. Note that despite the large and varied spiral patterns present, neither of the disks thicken noticeably nor do they evolve substantially in terms of their mass profiles even if they are run for times longer than $t_{2}$.

\section{RESULTS}

\subsection{The Provenance Bias of Radial Migration}

The non-axisymmetric patterns shown in Figure 1 lead to large exchanges of angular momentum and energy between disk particles. Since the mass profiles remain basically unchanged, a change in specific angular momentum, $L_{z}$, translates directly into a change in guiding center radius, $R_{g}$, defined by $L_{z}=R_{g} V_{\text {circ }}\left(R_{g}\right)$, where $V_{\text {circ }}^{2}=\partial \Phi / \partial \ln R$ and $\Phi$ is the gravitational potential on the disk plane. The larger the change in $R_{g}$, the farther a star migrates from its original ("birth") radius.

As discussed by Vera-Ciro et al. (2014), migrating stars are expected to exhibit a heavy vertical bias: the most extreme migrators are almost invariably those whose initial orbits do not take them far from the disk plane. This provenance bias of migrators is not surprising: kinematically cold stars with modest vertical excursions spend more time near the midplane and at the same galactocentric distance, and are thus able to couple more effectively to non-axisymmetric perturbations in the disk.
We illustrate this in Figure 3, where we have tracked the evolution in cylindrical radius (left panels) and height from the plane (right panels) of three particles in each simulation, chosen so that they share a common birth radius (i.e., $R_{g}\left(t_{0}\right)=5 \mathrm{kpc}$ ), but which either migrate inward (green curves) or outward (blue) by roughly $50 \%$, or stay put at their original radius (red). This figure illustrates clearly that migrating particles are preferentially those initially close to circular orbits and with small vertical excursions. Interestingly, we find the same qualitative result for all three simulated disks, despite the large differences in their spiral patterns.

The same conclusion is illustrated in Figure 4, where the left panels show, for all disk stars, the fractional change in guiding center radius, $\delta R_{g}=\ln R_{g}\left(t_{2}\right) / R_{g}\left(t_{0}\right)$, as a function of birth radius, $R_{g}\left(t_{0}\right)$. The colors encode the initial vertical velocity dispersion of migrators, in units of the average at the birth radius; i.e., $\sigma_{z}\left(R_{g}\left(t_{0}\right)\right)$. Blue colors denote kinematically cool stars whose vertical velocity dispersion are $\sim 20 \%$ below the mean; red corresponds to stars $\sim 15 \%$ hotter than the average.

The left panels of Figure 4 demonstrate that the efficiency of migration is a sensitive function of the initial $\sigma_{z}$ : extreme migrators are primarily those with small vertical motions at birth, a conclusion that applies equally well to all three simulations. Grand et al. (2016) reach the same conclusion in their study of a number of cosmological hydrodynamical simulations of Milky-Way-sized galaxies, so this finding seems robust: the provenance bias affecting stellar migration holds 


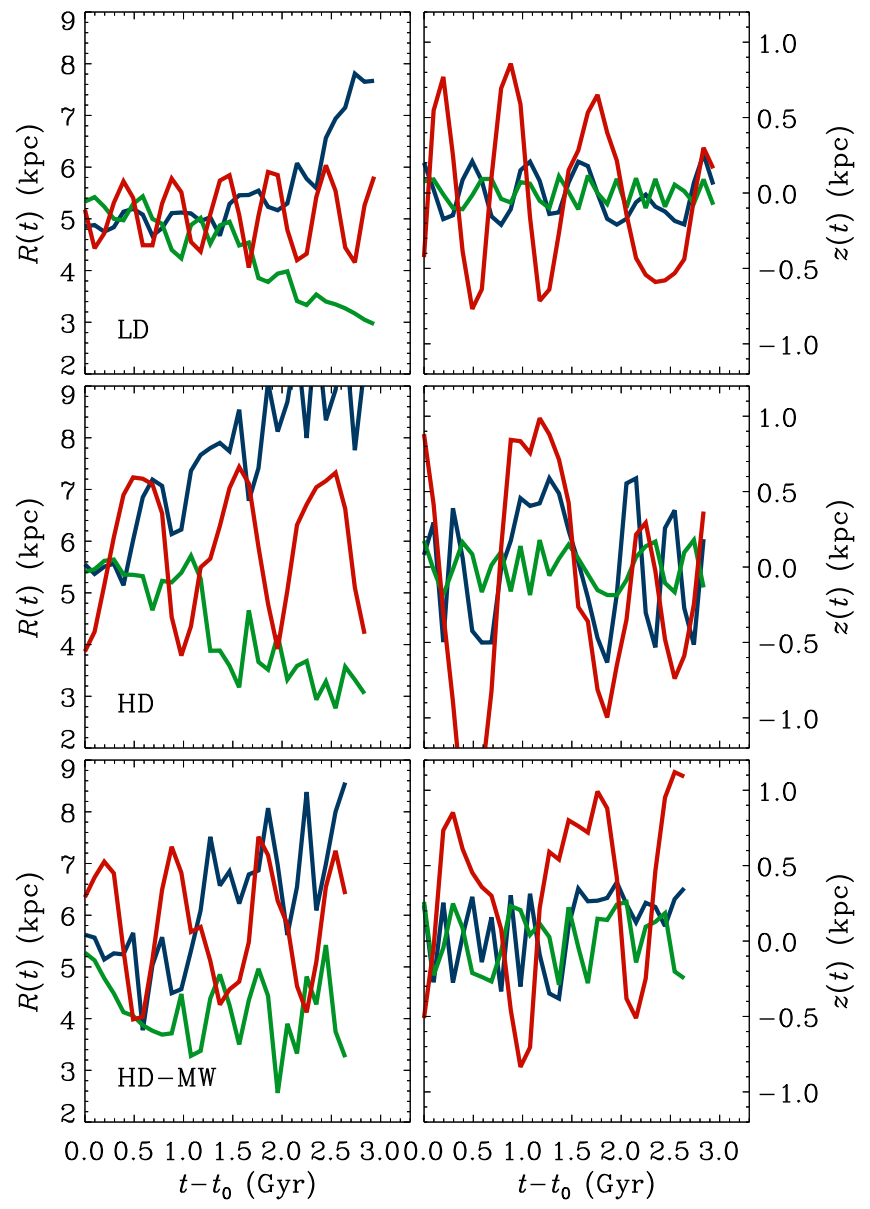

Figure 3. Orbits of three particles with similar guiding center radius at the initial time, $R_{g}\left(t_{0}\right)=5 \mathrm{kpc}$, but which migrate outward or inward by roughly $\sim 50 \%$ (blue/green curves, respectively.) The third (shown in red) does not show any substantial secular change in $R_{g}$. Left: cylindrical radius, $R$, as a function of time. Right: vertical displacement, $z$, (scale on right) as a function of time. Note the "provenance bias" of radial migrations, where extreme migrators are primarily particles initially on nearly circular orbits confined to the plane.

regardless of the morphology of spiral structure and strength of the non-axisymmetric perturbations.

\subsection{The Vertical Structure of Radial Migrators}

What is the vertical motion of migrators at their destination radii? This is shown in the right-hand panels of Figure 4, which are analogous to those on the left, but as a function of the final ("destination") guiding center radius, $R_{g}\left(t_{2}\right)$, and colored by the velocity dispersion in units of the average at the destination radius, $\sigma_{z}\left(R_{g}\left(t_{2}\right)\right)$. Inward migrators settle into orbits much more closely confined to the plane than the average at their new radii, whereas outward migrators end up with vertical velocity dispersions close to the average at their destination, except for a few radial strips, where migrators are slightly hotter than the pre-existing disk (see also Halle et al. 2015). This only happens for models where a strong bar dominates the kinematics at the final time; the vertically heated radial strips are consistent with the results expected for stars migrating inside the bar corotation radius, as discussed by Minchev et al. (2012).

The asymmetry between inward and outward migrators may be traced to the strong decline with a radius of $\sigma_{z}$ (see righthand panels of Figure 2) coupled with the vertical bias

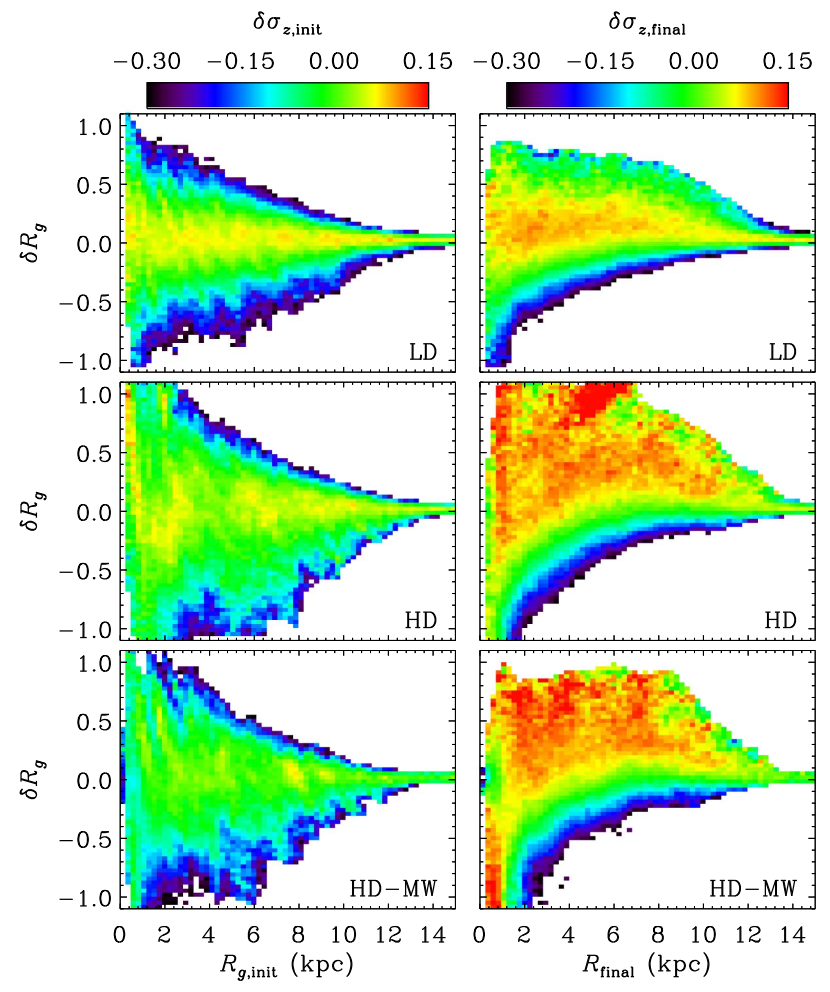

Figure 4. Left: fractional changes in guiding center radius $\left(\delta R_{g}=\ln R_{g}\left(t_{2}\right) / R_{g}\left(t_{0}\right)\right)$ as a function of the initial "birth radius," $R_{g}\left(t_{0}\right)$, colored by initial vertical velocity dispersion, $\sigma_{z}\left(t_{0}\right)$, expressed in units of the average value at the birth radius. Blue indicates stars with initial vertical excursions smaller than the average, red the opposite. Right: same as left, but as a function of the final guiding center "destination" radius, $R_{g}\left(t_{2}\right)$, and colored by the final vertical velocity dispersion, scaled to the average at the destination radius.

discussed above. The strong radial gradient implies that particles that move out arrive at radii, where the velocity dispersion is much lower than at their birth radius. Although they are initially biased and even cool down a little as they move out (Vera-Ciro et al. 2014), the gradient is so strong that their final velocity dispersions are much closer to the average in their new neighborhood than those that migrate inward. For the latter, the effects of the vertical bias are amplified by the larger velocity dispersion and higher surface densities of the inner regions: the vertical distribution of inward migrators becomes thinner and thinner the further in they drift.

As mentioned above, we note that, although outward migrators in general do not "heat" the disk vertically, there are some radii in which they do end up with higher-thanaverage velocity dispersion. An example is provided by the "red band" at $R_{g}\left(t_{2}\right) \sim 4-6 \mathrm{kpc}$ in the middle-right panel of Figure 4. Those radii correspond to regions in corotation with the strong bar that develop at late times in HD. This feature is thus likely caused by a bar resonance, and is actually not present at $t_{1}$, before the bar develops. (Similar patterns develop in the case of HD-MW, which also develops a bar at late times.) Indeed, no "red band" of vertically hot material is seen in the case of LD, where no bar develops at all. We should also note that these extreme migrators make up only a modest fraction of all stars at their destination radius, and their effect on the average kinematics there is negligible, as shown by the invariance of the $\sigma_{z}$ profiles shown in the right-hand panels of Figure 2. 
A study of the extent to which radial migration of stars is affected by their vertical motion has been presented in Solway et al. (2012). That study reports the response of two component stellar disks (a thin and a thick component) to perturbations induced by multiple spiral patterns with and without the presence of a bar. Although a quantitative comparison is difficult (our models have a single disk component) our results are qualitatively in agreement with theirs; i.e., thin-disk stars tend to be more affected by migration.

\subsection{Migration and Radial Gradients}

We explore now the effects of radial migration on preexisting radial gradients in the disks. Migration-led mixing is expected to blur initial trends, increasing the local dispersion and perhaps changing the shape of the local distribution of any stellar property tightly linked with its birth radius. One example of this is provided by the metallicity distribution function in the Galactic plane, which changes skewness inside/outside the solar circle, a feature probably caused by migration (Hayden et al. 2015).

In order to address such issues, we tag stars, at the initial time, $t=t_{0}$, according to the following relation.

$$
\langle[\mathrm{Fe} / \mathrm{H}]\rangle=0.12\left[8 \mathrm{kpc}-R_{g}\left(t_{0}\right)\right]
$$

with a Gaussian dispersion of $\sigma([\mathrm{Fe} / \mathrm{H}])=0.04$.

The slope of this equation is chosen so as to reproduce the radial dependence of the MAP peak radius reported by B16 for the Milky Way's $\alpha$-poor disk. We show this in the left panels of Figure 5, which display the initial metallicity gradient of each disk according to Equation (2), compared with the "peak radii" of five MAPs $([\mathrm{Fe} / \mathrm{H}]=-0.2,-0.1,0.0,0.1,0.2$, respectively). By construction, our initial disks have metallicities that match, at their birth radii, those of MAPs in the $\alpha$ poor ("thin") disk of the Galaxy.

How would the metallicity gradient evolve as a consequence of radial migration? This is shown in the middle panels of Figure 5, where we show the final gradient, computed at $t_{2}$. Although migration significantly blurs the initial trend, increasing the dispersion at a given radius, it does not affect the slope of the overall gradient much. Interestingly, it does not change the scale-height profile of the disk either, as may be seen in the righthand panels of Figure 5. Here the solid blue and red curves running vertically indicate the exponential scale height of stars at $t_{0}$ and $t_{2}$, respectively, as a function of $[\mathrm{Fe} / \mathrm{H}]$. Clearly, migration has had little effect on the overall disk scale height, measured either at a fixed radius, or at a fixed metallicity.

\subsection{Vertical Structure of Radial Migrators}

The right-hand panels of Figure 5 also illustrate one of the main differences between our models and the Galactic disk. Our models assume a constant initial scale height, which is basically unchanged by migration, and cannot therefore match, through the simple tagging proposed by Equation (2), the marked increase in thickness with decreasing metallicity observed in the Galaxy (colored symbols with error bars). The inability of radial migration to thicken the disk strongly suggests that the observed increase in Galactic disk thickness with decreasing metallicity (and increasing radius) is probably an intrinsic feature of the disk, and not a consequence of radial migration.
As mentioned in Section 1, one interesting property of Galactic MAPs is the presence of a variation of scale height with radius. These affect primarily MAPs in the low- $[\alpha / \mathrm{Fe}]$ ("thin") disk, whose scale heights increase monotonically with radius (B16). The gradients are such that, at fixed $[\mathrm{Fe} / \mathrm{H}]$, thindisk stars are thinner inside their "peak radius," and thicker outside. For example, Sun-like stars $([\mathrm{Fe} / \mathrm{H}]=0)$ "peak" at $\sim 8 \mathrm{kpc}$, where their exponential scale height is $h_{z} \sim 300 \mathrm{pc}$ : their scale height is a factor of $\sim 1.6$ thinner at $R \sim 4 \mathrm{kpc}$, and $\sim 1.6$ times thicker at $R \sim 14 \mathrm{kpc}$. Could this be the result of radial migration?

We address this question in Figure 6, where the thick solid curves display the radial profile of the scale height, $h_{z}(R)$, for five MAPs with metallicities selected between -0.2 $<[\mathrm{Fe} / \mathrm{H}]<0.2$ in bins 0.1 dex apart. To compare with the results reported by $\mathrm{B} 16$, we rescale each of the scale heights of the simulated MAPs to coincide with that observed in the Galaxy at their corresponding "peak" radius (indicated by the symbols). The profile of each MAP is further shifted vertically by an arbitrary amount for clarity. The color bands show the scale-height profile of the MAPs observed in the Milky Way (B16).

The main conclusion of this comparison is that radial migration, at least in our simulations, does not match the observed trends in the Galaxy. Migrators do thin down as they spread inward, but they do not thicken up as they move outward. As a result, although the vertical trend of a given MAP inside the peak radius agrees relatively well with the observed trend, outside that radius the simulated scale heights are systematically below those observed.

We emphasize that our results do not rule out that migration might play some role in the vertical trend, but, if it did, it would be responsible for the "thinning down" of a population inside its peak radius, rather than for its flaring outside of it, as is usually envisioned in scenarios, where the trend is driven by migration (Section 1). A further difficulty of such a scenario concerns the strong sensitivity of radial migration to the initial height of the population, and the fact that it would take longer for stars born in the outskirts of the Galaxy to move inward, given their long orbital times.

The latter is a strong constraint, given the strong dependence of migration efficiency with radius seen in Figure 4: whereas changes in a radius of $100 \%$ are not unusual at $\sim 4-5 \mathrm{kpc}$, they do not exceed $10 \%$ at $R \sim 12 \mathrm{kpc}$, mainly as a result of the increase in orbital time with radius. It is unclear then how stars in the Galaxy with, say, $[\mathrm{Fe} / \mathrm{H}] \sim-0.5$ - which would be born primarily at $R \sim 12 \mathrm{kpc}$ with a thickness of nearly $1 \mathrm{kpc}$ (B16) -would be able to spread efficiently around the Galaxy through radial migration, given their long orbital times and large thickness.

We end by noting that our models do not match the vertical structure of the Galaxy as a function of radius, neither at the beginning nor at the end of the simulations, so our conclusions are probably not the final word on this topic. Nevertheless, our discussion should serve to emphasize the critical role of provenance bias on radial migration, and the importance of carefully modeling the radial and vertical structure of the various Galactic populations when assessing the effects of migration. 


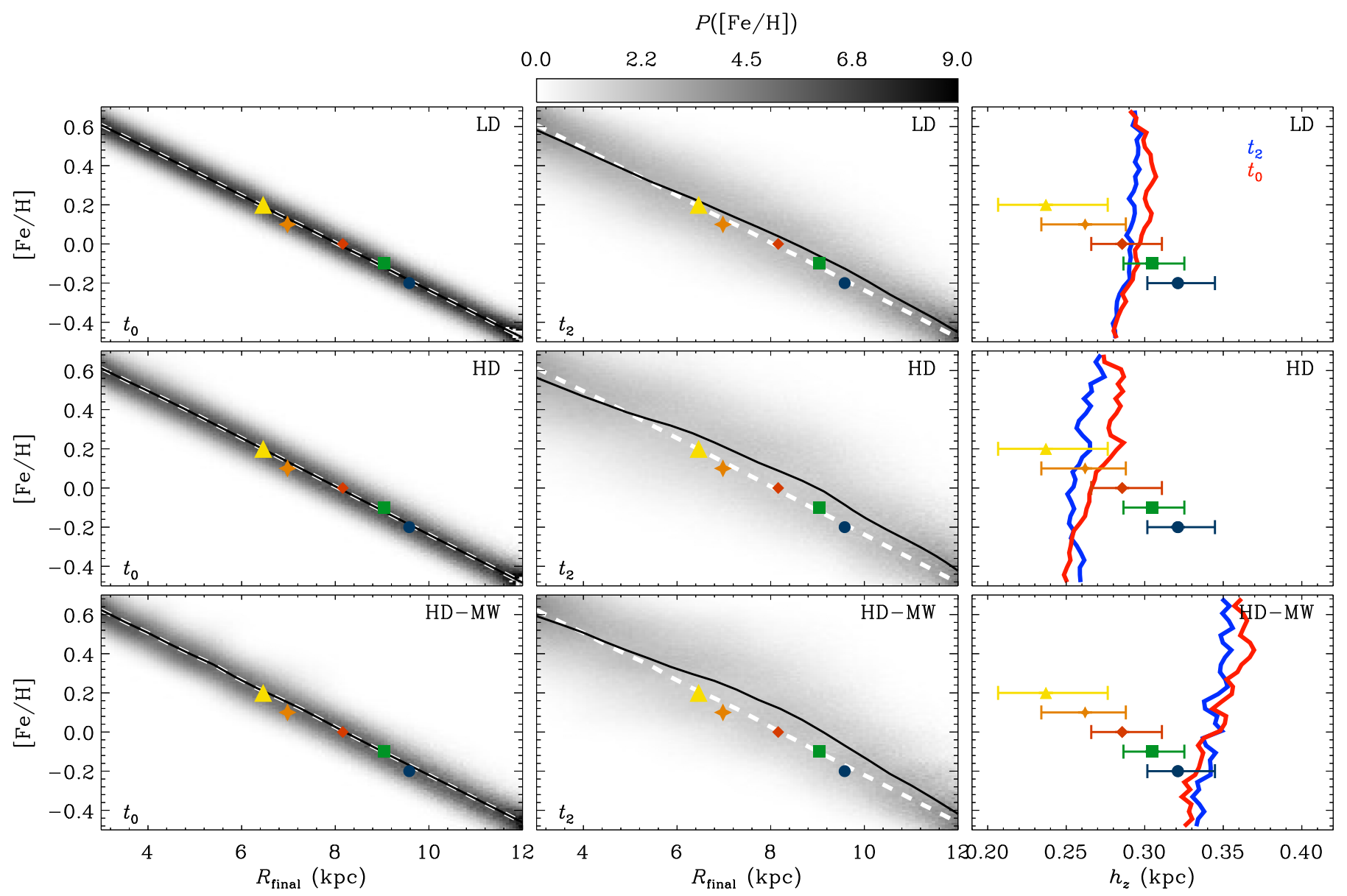

Figure 5. Left: metallicity gradient for the three simulated disks at $t_{0}$ (in gray), assumed to be $\langle[\mathrm{Fe} / \mathrm{H}]\rangle=0.12\left[8 \mathrm{kpc}-R_{g}\left(t_{0}\right)\right]$, with intrinsic widths of $\sigma([\mathrm{Fe} / \mathrm{H}])=0.04$. This is chosen to match the $[\mathrm{Fe} / \mathrm{H}]$ dependence of the "peak" radius of mono-abundance populations in the $\alpha$-poor Galactic disk. The peak radius of five MAPs with $[\mathrm{Fe} / \mathrm{H}]=-0.2,-0.1,0.0,0.1,0.2$, respectively, are shown by the colored symbols. Middle: same as left, but at $t_{2}$. A dashed line traces the initial gradient; the solid line tracks the median $[\mathrm{Fe} / \mathrm{H}]$ as a function of radius at $t_{2}$. Colored symbols are unchanged from the left panels. Right: exponential scale heights, $h_{z}$, as a function of $[\mathrm{Fe} / \mathrm{H}]$. Blue and red curves correspond to the initial and final scale height of the simulated disks, respectively. The initial scale height was assumed constant in our models, and remains basically unchanged, despite substantial radial migration. The Galactic disk, on the other hand, shows a strong trend of increasing thickness with decreasing metallicity, shown here at the "peak" radius of each MAP by the colored symbols with error bars. Data in all panels from B16.

\section{CONCLUSIONS}

We have used $N$-body models of isolated disk galaxies with realistic mass and circular velocity profiles to study the effects of radial migration. We focus on the vertical bias (which we term "provenance bias") favoring the migration of kinematically cold stars on nearly circular orbits confined to the disk (Vera-Ciro et al. 2014). The models contrast the effects of migration in a low-mass disk with weak, slowly evolving multi-armed spirals with those in heavy disks where the spiral patterns are stronger and with fewer arms, evolving quickly into a central bar.

Our main conclusions may be summarized as follows.

1. Provenance bias is present in all of our simulations, regardless of the nature of the spiral pattern. This bias implies that the efficiency of migration will depend sensitively on the thickness of a particular stellar population, a feature that must be taken carefully into account when modeling the effects of radial migration.

2. Provenance bias has a strong effect on the vertical structure of stars that have migrated away from their initial "birth" radius. Migrators are generally a kinematically colder subset for which the vertical velocity dispersion typically drops as they move out or increase as they move in. Their final structure is, therefore, heavily dependent on the variation of disk scale height with radius.

3. In our models, which feature a constant scale height and, thus, a strong radial gradient in $\sigma_{z}$, inward migrators become more and more heavily biased relative to the average population at their destination radius. Outward migrators, on the other hand, move to regions of lower $\sigma_{z}$ and become a closer match to the average population at their final radii.

4. In general, radial migrators thin down as they move in, but do not substantially thicken up as they move out, at least in disks like the ones we consider here. Radial migration alone thus does not provide a natural explanation for the monotonic increase with radius of the scale height of MAPs in the $\alpha$-poor Galactic disk reported by the APOGEE survey (B16).

Our results demonstrate that "provenance bias" plays a crucial role in the final vertical structure of stars that have migrated as a consequence of internal processes such as internally driven spiral patterns. Such migration does not lead, in general, to thicker disks, suggesting that the strong and monotonic increase of scale height seen in the $\alpha$-poor Galactic 

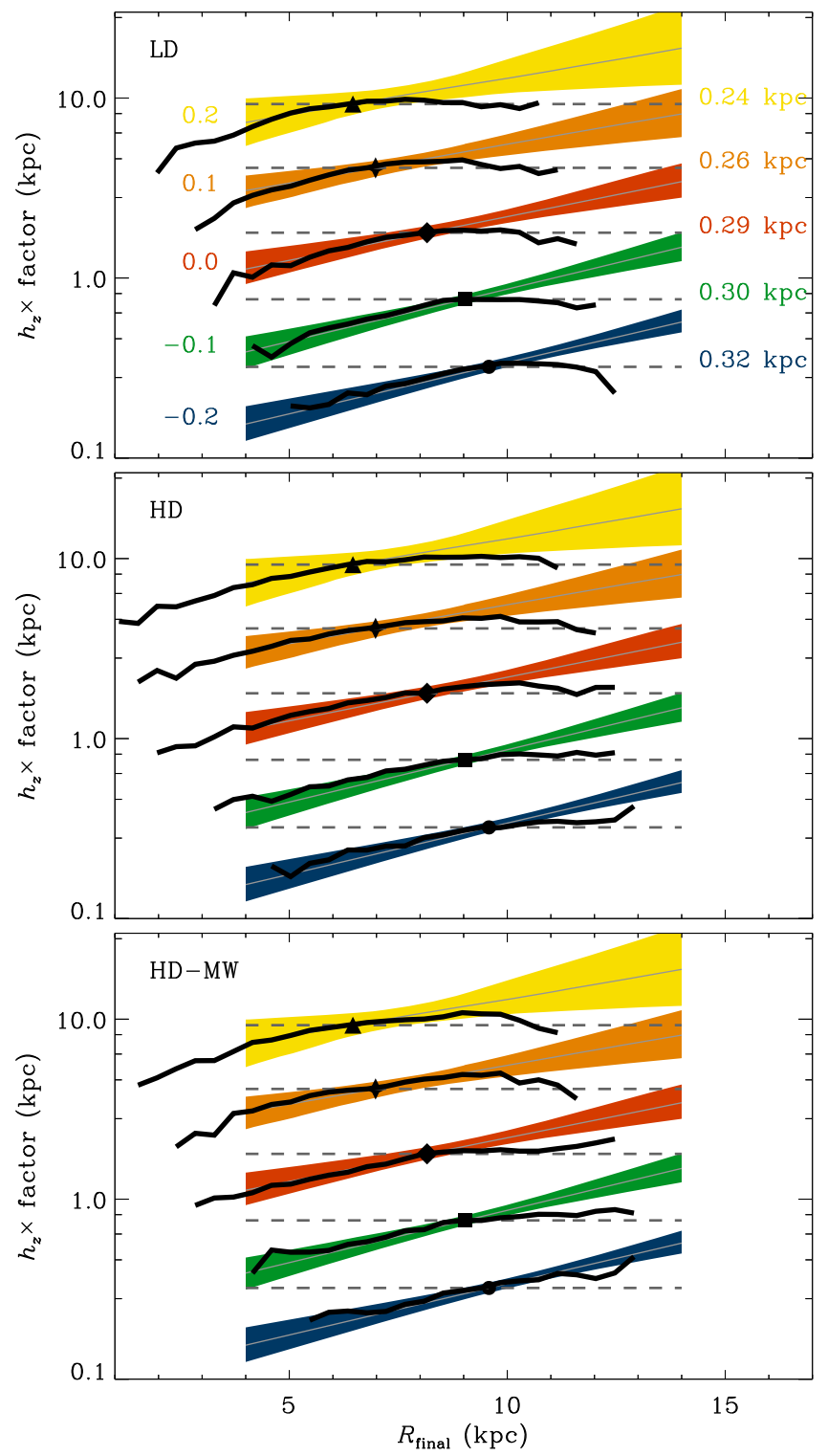

Figure 6. Radial dependence of the scale height of mono-abundance populations with $[\mathrm{Fe} / \mathrm{H}]=-0.2,-0.1,0.0,0.1,0.2$, respectively. Black curves indicate results for the simulated disks at $t_{2}$, colored bands show the results for the $\alpha$-poor Galactic disk from B16. Results for each MAP have been shifted vertically for clarity. The height of each simulated MAP has also been rescaled to match the observed value of the corresponding Galactic MAP at its "peak" radius. The latter are indicated by heavy symbols. Note that migrating stars thin down as they spread inward, but do not thicken up as they move outward. The observed radial trend is therefore unlikely to be caused by radial migration, particularly the outer "flaring" of each MAP.

disk has a different origin. Either the trend is inherent to the disk, or it was driven by external perturbations, such as accretion events and collisions with dark matter substructures.
A more definitive assessment of the importance of radial migration in the Galaxy will likely require cosmological models that are able to reproduce the observed trends in the radial and vertical distributions of the various mono-abundance populations that make up the disk in detail. Encouraging first steps have already been taken (see, e.g., Grand et al. 2016, and references therein), but a full understanding of the relative importance of internal and external mechanisms in shaping the Galactic disk seems still beyond reach.

This research has been partially funded by ATP NASA grant No NNX144AP53 and by the National Science Foundation under grants NSF AST-1211258 and NSF PHY11-25915. C.V.C. and J.F.N. acknowledge the hospitality of the Kavli Institute for Theoretical Physics at the University of California, Santa Barbara. E.D. gratefully acknowledges the support of the Alfred P. Sloan Foundation. Simulations have been run on the High Performance Computing cluster provided by the Advanced Computing Infrastructure (ACI) and Center for High Throughput Computing (CHTC) at the University of Wisconsin. We are grateful to Rob Grand for his very constructive comments and to Jo Bovy for kindly granting us access to his results.

\section{REFERENCES}

Bakos, J., Trujillo, I., \& Pohlen, M. 2008, ApJL, 683, L103 Bergemann, M., Ruchti, G. R., Serenelli, A., et al. 2014, A\&A, 565, A89 Bovy, J., Allende Prieto, C., Beers, T. C., et al. 2012, ApJ, 759, 131 Bovy, J., Rix, H.-W., Schlafly, E. F., et al. 2016, ApJ, 823, 30 (B16) D’Onghia, E., Vogelsberger, M., \& Hernquist, L. 2013, ApJ, 766, 34 Edvardsson, B., Andersen, J., Gustafsson, B., et al. 1993, A\&A, 275, 101 Grand, R. J. J., Springel, V., Gómez, F. A., et al. 2016, MNRAS, 459, 199 Halle, A., Di Matteo, P., Haywood, M., \& Combes, F. 2015, A\&A, 578, A58 Hayden, M. R., Bovy, J., Holtzman, J. A., et al. 2015, ApJ, 808, 132 Haywood, M. 2008, MNRAS, 388, 1175

Hernquist, L. 1990, ApJ, 356, 359

Hernquist, L. 1993, ApJS, 86, 389

Hernquist, L., \& Katz, N. 1989, ApJS, 70, 419

Kawata, D., Grand, R. J. J., Gibson, B. K., et al. 2016, MNRAS, 464, 702 Loebman, S. R., Debattista, V. P., Nidever, D. L., et al. 2016, ApJL, 818, L6 Loebman, S. R., Roškar, R., Debattista, V. P., et al. 2011, ApJ, 737, 8 Lynden-Bell, D., \& Kalnajs, A. J. 1972, MNRAS, 157, 1

Martinez-Medina, L. A., Pichardo, B., Moreno, E., \& Peimbert, A. 2016, MNRAS, 463, 459

Minchev, I., Famaey, B., Quillen, A. C., et al. 2012, A\&A, 548, A127

Minchev, I., Martig, M., Streich, D., et al. 2015, ApJL, 804, L9

Miranda, M. S., Pilkington, K., Gibson, B. K., et al. 2016, A\&A, 587, A10

Navarro, J. F., Abadi, M. G., Venn, K. A., Freeman, K. C., \& Anguiano, B. 2011, MNRAS, 412, 1203

Roškar, R., Debattista, V. P., \& Loebman, S. R. 2013, MNRAS, 433, 976

Roškar, R., Debattista, V. P., Quinn, T. R., Stinson, G. S., \& Wadsley, J. 2008, ApJL, 684, L79

Schönrich, R., \& Binney, J. 2009, MNRAS, 396, 203

Sellwood, J. A., \& Binney, J. J. 2002, MNRAS, 336, 785

Solway, M., Sellwood, J. A., \& Schönrich, R. 2012, MNRAS, 422, 1363

Vera-Ciro, C., \& D’Onghia, E. 2016, ApJ, 824, 39

Vera-Ciro, C., D’Onghia, E., Navarro, J., \& Abadi, M. 2014, ApJ, 794, 173 